\title{
SYMMETRY PREFERENCE AS A COGNITIVE BY-PRODUCT IN STARLINGS
}

\author{
by
}

\begin{abstract}
JOHN P. SWADDLE ${ }^{1)}$, JUDY P.K. CHE and R. EARL CLELLAND ${ }^{2)}$
(Institute for Integrative Bird Behavior Studies, Biology Department, College of William \& Mary, Williamsburg, VA 23187-8795, USA)
\end{abstract}

(Acc. 4-II-2004)

\begin{abstract}
Summary
There has been a great deal of interest in whether animals use trait symmetry as a visual cue to mediate behavioural interactions. In bilaterally symmetric traits, small asymmetries (termed fluctuating asymmetry) appear due to increased developmental stress and/or genes for poor developmental homeostasis. Hence, researchers have hypothesized that symmetry can reveal the developmental history and, perhaps, fitness of an individual and this is why symmetry preferences have been observed. However, an additional theory suggests that symmetry could be preferred merely because it represents the average expression of bilateral traits. Animals can learn to respond to signals by generalizing (or averaging) stimulus sets. As the average expression of a trait showing fluctuating asymmetry is zero asymmetry, theory predicts that animals could develop a symmetry preference as a by-product of learning. Here, we test this prediction empirically with European starlings (Sturnus vulgaris) and show that symmetry preferences can emerge as an outcome of generalized learning processes. Our results indicate that symmetry does not initially need to be associated with fitness to be an apparent cue in behavioural interactions and that symmetry preferences observed in nature could be independent of any putative fitness relationships.
\end{abstract}

\section{Introduction}

Animals, including humans, display preferences for symmetric cues and signals in some (Swaddle \& Cuthill, 1994; Møller \& Sorci, 1998; Morris \&

1) Corresponding author's e-mail address:jpswad@wm.edu

2) We thank Chuck Finneran and Doug Ruff for assistance with animal care. Paul Watson, Anne Hedrick, Martin Daly and an anonymous reviewer provided helpful comments on earlier drafts of the manuscript. This work was supported by the Thomas F. and Kate Miller Jeffress Memorial Trust and NSF (IBN-0133795). 
Casey, 1998; Rhodes et al., 1998) but not all cases (Swaddle \& Witter, 1995; Jablonski \& Matyjasiak, 1997; Tomkins \& Simmons, 1998; Breuker \& Brakefield, 2001). The most common explanation for a symmetry preference is that small amounts of (fluctuating) asymmetry (Ludwig, 1932) in bilateral traits reflect greater developmental stability and, therefore, could be a cue to increased genotypic (Møller \& Pomiankowski, 1993) or phenotypic fitness (Swaddle, 1997; Santos, 2001; Swaddle, 2003). Fluctuating asymmetry is the minor form of asymmetry that arises from random developmental accidents in traits that we would normally describe as being symmetric (Ludwig, 1932; Van Valen, 1962; Zakharov, 1981). Increasing developmental stress tends to increase expression of fluctuating asymmetry (Clarke, 1992; Møller \& Swaddle, 1997). Hence, it has been suggested that animals may prefer symmetric mates as symmetry could reveal fitness (Møller \& Swaddle, 1997).

As fluctuating asymmetry in bilaterally symmetric traits is diagnosed by a normal (or leptokurtic) distribution of signed asymmetries (i.e. left minus right values for a trait) that is centred around zero asymmetry, the average expression of a trait showing fluctuating asymmetry is symmetric (Palmer, 1994). Some theories of feature learning propose that animals may learn the characteristics of a set of signals by generalizing the learned set to the arithmetic mean (e.g. Langlois \& Roggman, 1990; Aydin \& Pearce, 1994). In subsequent recognition tasks, animals (including humans) give strongest responses to stimuli that most closely resemble generalized averages (Kalish \& Guttman, 1957; Blough, 1969; Strauss, 1979; Langlois \& Roggman, 1990; Dill \& Heisenberg, 1995; Enquist \& Johnstone, 1997; Jansson et al., 2002). Therefore, animals may develop symmetry preferences in traits that show fluctuating asymmetry because the arithmetic mean of these cues/signals is symmetric (Enquist \& Arak, 1994; Johnstone, 1994; Swaddle \& Cuthill, 1994; Enquist \& Johnstone, 1997).

Initial explorations of this prediction are consistent with this view (Jansson et al., 2002). Jansson and colleagues trained chickens (Gallus gallus domesticus) to peck at asymmetric crosses that were either left- or right-biased. In non-reinforced probe trials, the birds showed preferences for novel symmetric crosses that were the arithmetic mean of the training stimuli. However, these data cannot be exclusively interpreted as support for generalizing theories as (i) the experimenters used symmetric images in the initial shaping procedures of the experiment, which could have affected symmetry preferences in the later probe trials; and (ii) the symmetric probe images were 
novel to the birds, whereas the alternative images in probe trials were familiar. Hence, symmetry was confounded with image novelty, and 'novelty' is known to affect preference tests in experimental situations (e.g. Vallortigara et al., 1994; Jones et al., 1996; Nowicki et al., 2001). Here, we report an experiment in which we have minimized such effects and tested experimentally whether a common bird, the European starling (Sturnus vulgaris), can develop a symmetry preference as a by-product of learning a set of images that vary in asymmetry.

Specifically, we constructed learning trials in which starlings learned to feed from dishes that displayed (i) left-biased asymmetric images, (ii) rightbiased asymmetric images, or (iii) both left- and right-biased images. We then examined birds' preferences in a probe test for novel symmetric and novel asymmetric images versus control birds that were not trained. If the birds generalize during the learning part of the experiment, asymmetric trained birds should display a relative preference for novel asymmetric dishes (biased in the direction of their learning set), whereas birds trained with both left- and right-biased images should prefer the novel symmetric dishes. We hypothesized that starlings will show some degree of stimulus generalization in test trials and, specifically, that generalization will lead to symmetry preferences in the birds exposed to both left- and right-biased images in learning trials.

\section{Methods}

We used thirty-four wild-caught adult European starlings of both sexes in this study. Two birds died of natural causes before the completion of the experiment, and four did not complete the experiment due to unresponsiveness in the experimental arena. Therefore, twentyeight birds completed the experiment. The birds were housed in small groups (three or less) in cages $(90 \times 40 \times 40 \mathrm{~cm})$ with perches and drinking water on a $8: 16$ hour light: dark photoperiod so that birds did not undergo gonadal hypertrophy or moult (Dawson \& Goldsmith, 1983). The starlings had ad libitum access to chick crumbs, except on experimental days (four or five days per week) when food was removed during the previous night.

Experimental trials began one to two hours after 'dawn' (i.e. when the lights turned on in the animal rooms). The experiment was conducted in a room (approximately $4 \times 3 \times 3 \mathrm{~m}$ ) where we marked on the floor a $1 \mathrm{~m}^{2}$ grid with 100 equally spaced locations. $1.5 \mathrm{~m}$ tall perches were placed at opposite corners just outside the grid so birds could easily view the grid from above. We placed twenty clear petri dishes at randomly selected locations on the grid for each trial. Each dish contained a small compartment into which we placed the same amount of food (approximately $0.4 \mathrm{~g}$ ). The compartment was constructed so that food did not spill into the rest of the dish and obscure the image in any way. For ten dishes, we placed chick 


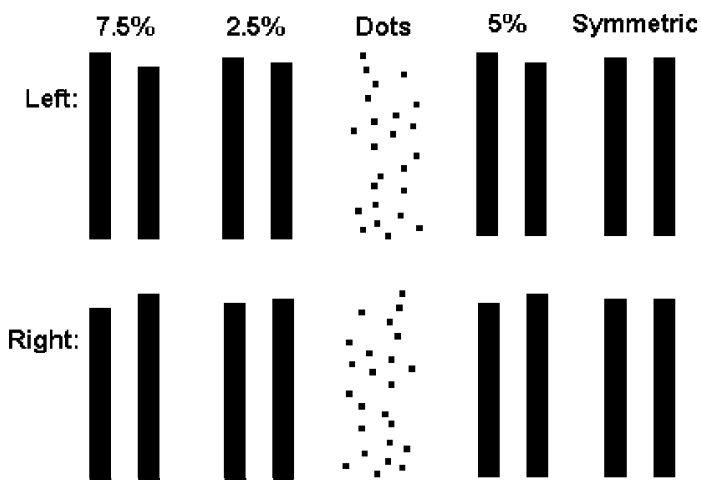

Fig. 1. Examples of the bar patterns used in learning and test trials. Birds were trained with $7.5 \%$ and $2.5 \%$ asymmetric images as $\mathrm{S}^{+}$(positive reinforcement), versus dot images as $\mathrm{S}^{-}$ (negative reinforcement). 5\% asymmetric and symmetric images were used in test trials. See Methods for details of treatment groups.

food that had been sprayed with a $30 \%$ quinine hydroxide solution and then dried. This food is distasteful to this birds but not harmful (Forsman \& Merilaita, 1999). In the remaining ten dishes we placed chick food that had been sprayed with distilled water and then dried (control food). By placing different categories of printed stimulus images under the 'control' versus the 'quinine' dishes we associated positive $\left(\mathrm{S}^{+}\right)$and negative $\left(\mathrm{S}^{-}\right)$reinforcement, respectively, with different image categories.

The $\mathrm{S}^{+}$stimuli were monochromatic, paired-bar patterns (dark bars on a light background). There were four sets of patterns: $2.5 \%$ left-biased (in which the left bar was $2.5 \%$ taller than the right), $2.5 \%$ right-biased, $7.5 \%$ left-biased, and $7.5 \%$ right-biased. All four sets had the same overall mean bar size. However, within each of the four sets there was variation in bar sizes to give ten distinct bar patterns (i.e. there were ten representations of $2.5 \%$ leftbiased, ten $2.5 \%$ right-biased, ten $7.5 \%$ left-biased, and ten $7.5 \%$ right-biased patterns). From previous experiments, we know that starlings categorize such patterns as asymmetric and can discriminate $2.5 \%$ asymmetry from $7.5 \%$ asymmetry (Swaddle, 1999). $\mathrm{S}^{-}$stimuli were a set of 20 asymmetric random dot patterns (20-36 dark dots on a light background). All images were printed at $1200 \mathrm{dpi}$ on white letter paper (approx. $5 \times 2.5 \mathrm{~cm}$ ) and laminated (Fig. 1).

The starlings were randomly allocated to four groups for learning trials $(N=7$ in each group). (1) Left birds were trained to peck at five $2.5 \%$ and five $7.5 \%$ left-biased $\mathrm{S}^{+}$images versus ten $\mathrm{S}^{-}$images. (2) Right birds were trained to peck at five $2.5 \%$ and five $7.5 \%$ rightbiased images versus ten $\mathrm{S}^{-}$images. (3) Both birds were trained to peck at two (or three) $2.5 \%$ left- and three (or two) 2.5\% right-biased images and two (or three) $7.5 \%$ left- and three (or two) $7.5 \%$ right-biased $\mathrm{S}^{+}$images versus ten $\mathrm{S}^{-}$images. (4) Control birds did not receive any learning trials but were tested for spontaneous symmetry preferences in non-reinforced test trials (described below). For all learning treatment groups (i.e. groups 1, 2, and 3), images were randomly selected from the larger stimulus set for each trial.

A learning trial consisted of placing the appropriate images under the twenty dishes and releasing the bird into the room. We recorded activity with a digital video camera. For each trial, we noted the location of each dish from which the starling fed. We ignored exploratory pecking when food did not enter the mouth. Repeated visits to a particular dish were recorded 
as separate preferences as long as there was an intervening search or a departure from the grid and there was food left in the compartment of the dish. In the vast majority of cases, starlings did not consume all of the food in a dish before searching for another dish. This procedure maintained a constant probability of visitation for each type of image $\left(\mathrm{S}^{+}\right.$or $\left.\mathrm{S}^{-}\right)(c f$. Osorio et al., 1999). Trials lasted 40 minutes or until the bird had eaten from eight dishes. Birds were trained and tested in blocks of four, one at a time and in random order. From these data, we calculated the percentage of feeding visits to $\mathrm{S}^{+}$dishes (i.e. those with the bar images) by each bird in each trial.

Each bird from groups 1,2, and 3 was tested after completing three consecutive learning trials with a $75 \%$ (or greater) selection of $\mathrm{S}^{+}$dishes. On average $( \pm S E M)$, this took 8.71 $( \pm 0.85)$ trials and all birds satisfied the learning criteria by trial 16. Control birds were tested without any learning trials. Testing trials were similar to learning trials except that test trials employed (palatable) control food exclusively, and birds were exposed to novel sets of images. These images had the same properties as $\mathrm{S}^{+}$learning stimuli, except they differed in their extent of bar asymmetry. (1) Left birds experienced ten 5\% asymmetric left-biased bar patterns and ten symmetric bar patterns. (2) Right birds experienced ten $5 \%$ asymmetric rightbiased bar patterns and ten symmetric bar patterns. (3) Both and Control birds experienced five 5\% asymmetric left-biased, five 5\% asymmetric right-biased, and ten symmetric bar patterns. We recorded data identical to that recorded during the learning trials, noting the percentage of feeding visits to symmetrical images out of the eight dishes selected. Hence, we tested for bird's preferences for novel images that either fit or did not fit the arithmetic mean of learning images. Only in the Both treatment is the arithmetic mean symmetric. We also tested for spontaneous symmetry/asymmetry preferences in Control birds.

All birds were retained in captivity following this study for future experiments. Our protocol is in compliance with the Animal Welfare Act and was approved by our Institutional Animal Care and Use Committee \#0126. All statistical analyses employed two-tailed tests of significance using SPSS 11.5 for Windows. We examined the residuals from all analyses to ensure that our data met the assumption of normality for parametric tests, and the assumption of homogeneity of variance for ANOVA.

\section{Results}

\section{Test of stimulus generalization}

For the birds who experienced learning trials (i.e. birds from groups 1, 2 and 3 ), birds were significantly more likely than chance to visit and feed from dishes displaying images that were the average of their learning set (onesample $t$-test against prediction of random (0.5) feeding visits to average of learning images, $t_{20}=2.49$, two-tailed $p=0.022$; mean $( \pm S E M)$ frequency of feeding visits to average dishes $=0.570( \pm 0.028)$ ). Therefore, in general, there is support for the birds generalizing their stimulus sets from the learning trials. Moreover, there was no significant difference in the extent of this generalization effect among treatment groups (one-way ANOVA: $\left.F_{2,18}=2.16, p=0.145\right)$. 


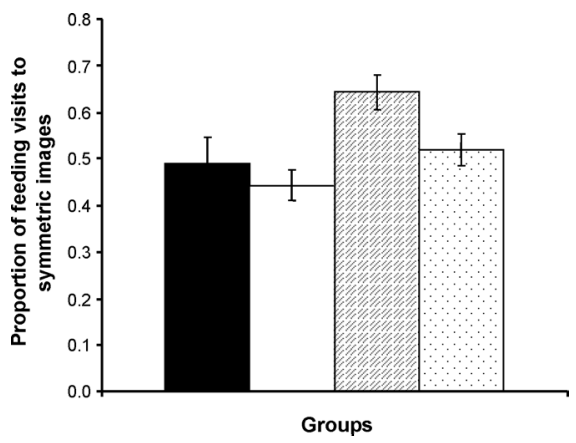

Fig. 2. Mean ( $\pm S E M)$ proportion of feeding visits to symmetric images in test trials across the three treatment groups. Left birds (trained on left-biased bars) represented by black bar, Right birds (trained on right-biased bars) represented by hollow bar, Both birds (trained on both left- and right-biased bars) represented by striped bar, Control birds (not trained) represented by the stippled bar. Both birds were more likely to visit and feed from dishes displaying symmetric images than the other groups. 0.5 indicates random visits to either symmetric or asymmetric dishes.

Test of symmetry preferences

As predicted, we found a significant difference among all treatment groups in feeding visits to symmetric versus asymmetric images in test trials (one-way ANOVA: $F_{3,27}=3.897$, two-tailed $p=0.021$; Fig. 2 ). A priori contrasts revealed that Both birds were more likely to feed from dishes in test trials containing symmetric images than Left and Right birds combined ( $p=$ 0.005), and more likely to feed from symmetric dishes than Control birds ( $p=0.041$ ). However, Left and Right birds were not more likely to eat from asymmetric dishes than Control birds $(p=0.329)$ even though Control birds did not show a spontaneous preference for either symmetry or asymmetry in their test trials (one-sample $t$-test against prediction of random $(0.5)$ feeding visits to symmetric test images, $t_{6}=0.578$, two-tailed $p=0.585$ ).

\section{Discussion}

Our results are consistent with the explanation that starlings engage in a learning process that leads to stimulus generalization and, in test trials, birds subsequently prefer test images that are closer to the arithmetic mean of the learning set. Specifically, our study provides some empirical support for theoretical arguments that symmetry preferences can arise as a by-product 
of cognitive processes, as the arithmetic mean of traits showing fluctuating asymmetry is zero asymmetry (Enquist \& Arak, 1994; Johnstone, 1994; Swaddle \& Cuthill, 1994; Enquist \& Johnstone, 1997). Untrained birds did not have a preference for symmetry, as is consistent with previous data from starlings (Swaddle, 1999; Swaddle \& Pruett-Jones, 2001; Swaddle \& Ruff, 2004), yet birds trained to detect a set of asymmetric images that were not side-biased developed a symmetry preference. This result suggests a mechanism by which symmetry preferences can initially arise in a population independent of a putative relationship between asymmetry and genotypic (Møller \& Pomiankowski, 1993) or phenotypic fitness (Swaddle, 1997, 2003; Santos, 2001). Once sexual selection has acted upon this bias for symmetry, it is likely that an asymmetry-fitness relationship would evolve (Kokko et al., 2002, 2003). However, in the initial stages of this process, a symmetry preference can be established in a population without a relationship between asymmetry and fitness. Therefore, a symmetry preference need not be indicative of a current asymmetry-fitness relationship.

A curious pattern in our data is that birds in the Left and Right treatments did not prefer the asymmetric images in test trials, as would be predicted by stimulus generalization, though the weak trend was in the correct direction. However, it is important to note that our finding of symmetry preferences in Both birds does not exclude the possibility that other cognitive mechanisms also influence signal preferences. It is clear that birds can learn image differences based on recognition of individual images (Pearce, 1989) and that recognition of individual images may affect successful categorization of symmetric images by starlings (Swaddle \& Pruett-Jones, 2001). We predict that when signals have low variability individual image recognition should be more prevalent, as there are fewer signal images to store and recall. As Both birds viewed a greater variance in their learning stimuli (i.e. both left- and right-biased images as opposed to just one of these categories), we hypothesize that they should be more likely to use stimulus generalization mechanisms in learning their set of stimuli. Left and Right birds may (relative to Both birds) rely more on individual image recognition mechanisms and, hence, show a weaker fit with generalization predictions. This hypothesis could be tested in subsequent experiments. It is interesting to note that the Both treatment more accurately represents the distributions of asymmetries a birds would experience in nature - a bird would not normally experience population side-biased asymmetries when viewing bilaterally symmetric traits. 
In a similar manner that systematic studies of pre-existing sensory biases have affected how we study and interpret sexual signals (Endler \& Basolo, 1998), the data from our study, combined with similar findings in chickens (Jansson et al., 2002), indicate that we need to review our interpretation of fluctuating asymmetry cues. It could be that some of the symmetry preferences reported in the literature originate from general cognitive by-products of learning. If this hypothesis is true, we would expect to see symmetry preferences in nature without an apparent asymmetry-fitness relationship (if sexual selection has not had sufficient time to drive a correlation between asymmetry and fitness). Interestingly, for the two (nonhuman) species we are aware of in which there is unequivocal evidence of symmetry preferences among conspecifics (zebra finches Taeniopygia guttata and swordtail fish Xiphophorus cortezi) neither have reported a relationship between natural levels of asymmetry and fitness parameters (Swaddle \& Cuthill, 1994; Morris \& Casey, 1998). For the zebra finch, in a series of unpublished observational studies, we have not found any evidence of the predicted negative relationship between feather asymmetry and fitness metrics (J.P. Swaddle, unpubl. data). Therefore, our current data suggest that symmetry preferences can result from a by-product of learning mechanisms and that documentation of a symmetry preference is not necessarily indicative of an asymmetryfitness relationship.

\section{References}

Aydin, A. \& Pearce, J.M. (1994). Prototype effects in categorization by pigeons. — J. Exp. Psychol.: Anim. Behav. Process 20, p. 264-277.

Blough, D.S. (1969). Generalization gradient shape and summation in steady-state tests. J. Exp. Anal. Behav. 12, p. 91-104.

Breuker, C.J. \& Brakefield, P.M. (2001). Female choice depends on size but not symmetry of dorsal eyespots in the butterfly Bicyclus anynana. - Proc. R. Soc. Lond. B 269, p. 1233-1239.

Clarke, G.M. (1992). Fluctuating asymmetry: A technique for measuring developmental stress of genetic and environmental origin. - Acta Zool. Fennica 191, p. 31-35.

Dawson, A. \& Goldsmith, A.R. (1983). Plasma prolactin and gonadotrophins during gonadal development and the onset of photorefractoriness in male and female starlings (Sturnus vulgaris) on artificial photoperiods. - J. Endocrinol. 97, p. 253-260.

Dill, M. \& Heisenberg, M. (1995). Visual pattern memory without shape recognition. — Phil. Trans. R. Soc. Lond. B 349, p. 143-152.

Endler, J.A. \& Basolo, A.L. (1998). Sensory ecology, receiver biases and sexual selection. Trends Ecol. Evol. 13, p. 415-420. 
Enquist, M. \& Arak, A. (1994). Symmetry, beauty and evolution. — Nature 372, p. 169-172. — — \& Johnstone, R.A. (1997). Generalization and the evolution of symmetry preferences. — Proc. R. Soc. Lond. B 264, p. 1345-1348.

Forsman, A. \& Merilaita, S. (1999). Fearful symmetry: pattern size and asymmetry affects aposematic signal efficacy. - Evol. Ecol. 13, p. 131-140.

Jablonski, P.G. \& Matyjasiak, P. (1997). Chaffinch (Fringilla coelebs) epaulette display depends on the degree of exposure but not symmetry of intruder's epaulettes. - Behaviour 134, p. 1115-1121.

Jansson, L., Forkman, B. \& Enquist, M. (2002). Experimental evidence of receiver bias for symmetry. - Anim. Behav. 63, p. 617-621.

Johnstone, R.A. (1994). Female preference for symmetrical males as a by-product of selection for mate recognition. - Nature 372, p. 172-175.

Jones, R.B., Larkins, C. \& Hughes, B.O. (1996). Approach/avoidance responses of domestic chicks to familiar and unfamiliar video images of biologically neutral stimuli. - App. Anim. Behav. Sci. 48, p. 81-98.

Kalish, H.I. \& Guttman, N. (1957). Stimulus generalization after equal training on two stimuli. - J. Exp. Psychol. 53, p. 139-144.

Kokko, H., Brooks, R., Jennions, M.D. \& Morley, J. (2003). The evolution of mate choice and mating biases. - Proc. R. Soc. Lond. B 270, p. 653-664.

— — , — - McNamara, J.M. \& Houston, A.I. (2002). The sexual selection continuum. Proc. R. Soc. Lond. B 269, p. 1331-1340.

Langlois, J.H. \& Roggman, L.A. (1990). Attractive faces are only average. — Psychol. Sci. 1, p. 115-121.

Ludwig, W. (1932). Das rechts-links problem im tierreich und beim menschen. — SpringerVerlag, Berlin.

Møller, A.P. \& Pomiankowski, A. (1993). Fluctuating asymmetry and sexual selection. Genetica 89, p. 267-279.

— — \& Sorci, G. (1998). Insect preference for symmetrical artificial flowers. — Oecologia 114 , p. 37-42.

— — \& Swaddle, J.P. (1997). Asymmetry, developmental stability and evolution. — Oxford University Press, Oxford.

Morris, M.R. \& Casey, K. (1998). Female swordtail fish prefer symmetrical sexual signal. — Anim. Behav. 55, p. 33-39.

Nowicki, S., Searcy, W.A., Hughes, M. \& Podos, J. (2001). The evolution of bird song: male and female response to song innovation in swamp sparrows. - Anim. Behav. 62, p. 1189-1195.

Osorio, D., Jones, C.D. \& Vorobyev, M. (1999). Accurate memory for color but not pattern contrast in chicks. - Curr. Biol. 9, p. 199-202.

Palmer, A.R. (1994). Fluctuating asymmetry analyses: A primer. — In: Developmental instability: Its origins and evolutionary implications (T.A. Markow, ed.). Kluwer, Dordrecht, p. 335-364.

Pearce, J.M. (1989). The acquisition of an artificial category by pigeons. - Q. J. Exp. Psychol. 41, p. 381-406.

Rhodes, G., Proffitt, F., Grady, J.M. \& Sumich, A. (1998). Facial symmetry and the perception of beauty. - Psychonom. Bull. Rev. 5, p. 659-669.

Santos, M. (2001). Fluctuating asymmetry is nongenetically related to mating success in Drosophila buzzatii. — Evolution 55, p. 2248-2256. 
Strauss, M. (1979). Abstraction of prototypical information by adults and 10-month-old infants. - J. Exp. Psychol: Hum. Learn. Mem. 5, p. 618-632.

Swaddle, J.P. (1997). Within-individual changes in developmental stability affect flight performance. - Behav. Ecol. 8, p. 601-604.

- - (1999). Limits to length asymmetry detection in starlings: implications for biological signalling. - Proc. R. Soc. Lond. B 266, p. 1299-1303.

— - (2003). Fluctuating asymmetry, animal behavior, and evolution. — Adv. Study Behav. 32 , p. 169-205.

- — \& Cuthill, I.C. (1994). Female zebra finches prefer males with symmetric chest plumage. - Proc. R. Soc. Lond. B 258, p. 267-271.

- — \& Pruett-Jones, S. (2001). Experimental investigation of the acquisition of learned symmetry discrimination in starlings. - Am. Nat. 158, p. 300-307.

— — \& Ruff, D.A. (2004). Starlings have difficulty in detecting dot symmetry: implications for studying fluctuating asymmetry. - Behaviour 141, p. 29-40.

_ — \& Witter, M.S. (1995). Chest plumage, dominance and fluctuating asymmetry in female starlings. - Proc. R. Soc. Lond. B 260, p. 219-223.

Tomkins, J.L. \& Simmons, L.W. (1998). Female choice and manipulations of forceps size and symmetry in the earwig Forficula auricularia L. - Anim. Behav. 56, p. 347-356.

Van Valen, L. (1962). A study of fluctuating asymmetry. — Evolution 16, p. 125-162.

Vallortigara, G., Regolin, L. \& Zanforlin, M. (1994). The development of responses to novelcoloured objects in male and female domestic chicks. - Behav. Proc. 31, p. 219-230.

Zakharov, V.M. (1981). Fluctuating asymmetry as an index of developmental homeostasis. - Genetika 13, p. 241-256. 